\title{
HISTOLOGICAL EXAMINATION OF THE VENTRICULAR MYOCARDIUM IN 62 CASES OF CONGENITAL HEART DISEASE*
}

\author{
BY \\ JENNIFER M. BROWN \\ From the Department of Pathology, Adelaide Children's Hospital, South Australia \\ Received September 2, 1963
}

A search through the journals and standard texts (e.g. Gould, 1960) yields plenty of data on the relation of hypertrophy of the four heart chambers to various structural cardiac defects, but evidence on the presence or absence of irreversible damage to the muscle fibres, in the form of necrosis or replacement fibrosis, is singularly scanty. With the increasing importance of surgery in the treatment of congenital cardiac anomalies, information on this aspect would obviously be of value, and with this in mind a series of 62 hearts showing various congenital structural deformities was investigated.

\section{MATERial AND Methods}

An unselected series of hearts, which showed congenital abnormalities, was preserved over the course of seven years from routine necropsies performed at the Adelaide Children's Hospital. A complete slice was taken from both ventricles and the interventricular septum at about the mid-ventricular level, well away from any ventricular septal defect when present. It was thought that this would provide a good representative section and one suitable for comparison of the lesions from a topographical point of view. The material was then processed through a double impregnation method using 1 per cent celloidin in methyl-benzoate and the sections stained with hæmatoxylin and eosin and with hæmatoxylin and van Gieson's mixture. A smaller piece of one or both ventricles was taken for frozen sectioning and stained with Fettrot 7B to demonstrate lipoid.

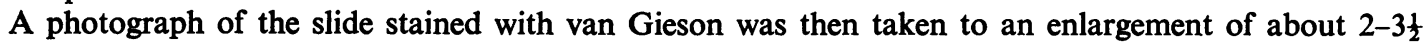
times, depending on the initial size of the heart (see Fig. 1). This was used to map out the location and extent of any histological abnormalities.

Microscopical Examination. An attempt was made to identify on the slide the right from the left ventricle. This was not always easy to do, particularly in the rare case where the ventricles were transposed, but the photographs and the post-mortem record of the macroscopical appearance of the heart proved of considerable help. The sections stained with van Gieson were then divided, by means of inked lines, into segments of a size suitable for careful microscopical inspection, and these divisions were duplicated both on the preparations stained with hæmatoxylin and eosin and on the projection print. The van Gieson sections were then examined with a large hand lens, and areas of fibrosis marked on the corresponding areas in the photographs. This was then confirmed using the low-power lens of the microscope, and other areas were added, too small to be picked up with the hand lens. This was done systematically, one area at a time. The hæmatoxylin and eosin preparation was then carefully examined under the microscope, area by area; any further abnormality discovered was recorded on the photograph. The sections stained with Fettrot 7B were then examined for evidence of fatty change.

* This study was aided by a grant from the National Heart Foundation of Australia. 


\section{RESULTS}

Sex Distribution. Thirty-six cases were female, twenty-five were male, and one unknown.

Age at Death. The length of survival after birth ranged from 2 days to 27 months (2 cases unknown). By the age of 3 weeks, 22 were dead. By 6 months, 47 had died. Of the remaining 13 cases, 6 lived beyond one year.

Types of Malformation. An attempt was made to group the abnormalities into types, but this proved difficult owing to the great variety of anomalies present. As 39 different combinations existed in the 62 hearts studied, an attempt was made to classify the lesions as near as possible into a lesser number of more basic types, resulting in ten varieties (first column in the Table).

Weight of the Heart. In 26 cases, the weight of the heart was not recorded, but of the remaining 36 cases, only one showed no increase above the average normal weight. The others all showed increases ranging up to a maximum increase of 250 per cent (Coppoletta and Wolbach, 1933).

Cyanosis. Seventeen cases did not show cyanosis at any time; 44 did show cyanosis, 17 being cyanosed continually from birth, 2 intermittently from birth, and 1 occasionally from birth; in 14, the onset of cyanosis was variable (from 3 days of age up to 26 months); in 10 the duration was not known. In one, no history was available.

Histopathological Findings. Here, again, classification into basic types proved difficult owing to the diversity of combinations of pathological changes ranging in nature from acute myocardial necrosis to calcification, and in extent from one small patch to more widespread involvement. However, some attempt has been made to classify the findings into broad pathological types (3rd, 4th, and 5th columns in the Table), relating them to the type of malformation. Twenty cases were normal histologically (apart from hypertrophy); 37 showed minor changes ranging from generalized sub-endocardial fibrosis without myocardial degeneration (Fig. 2) to mild patchy acute necrosis and/or fibrosis; and 5 showed severe changes, to be described further.

Fibre Hypertrophy. The left ventricle

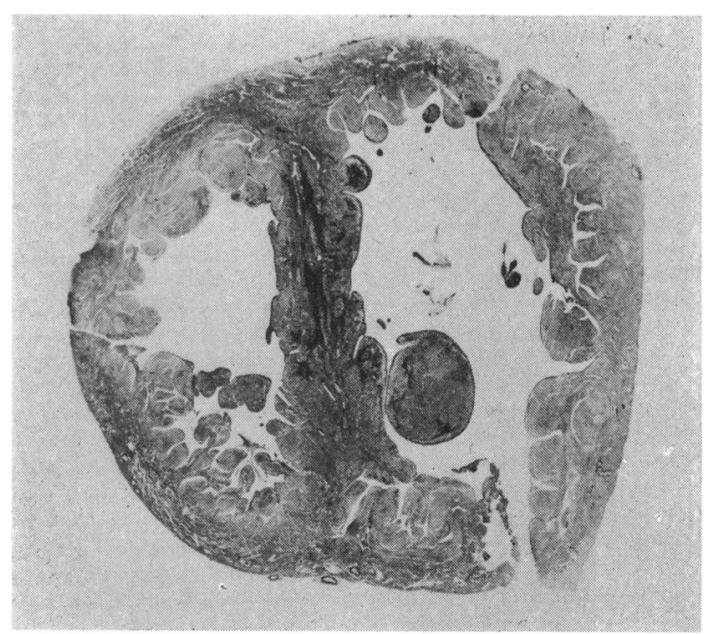

Fig. 1.-Case 2. An example of the large complete slice of the ventricles used in this study. The dark areas, most marked in the septum, represent fibrosis. (van Gieson. $\times 1 \cdot 7$.)

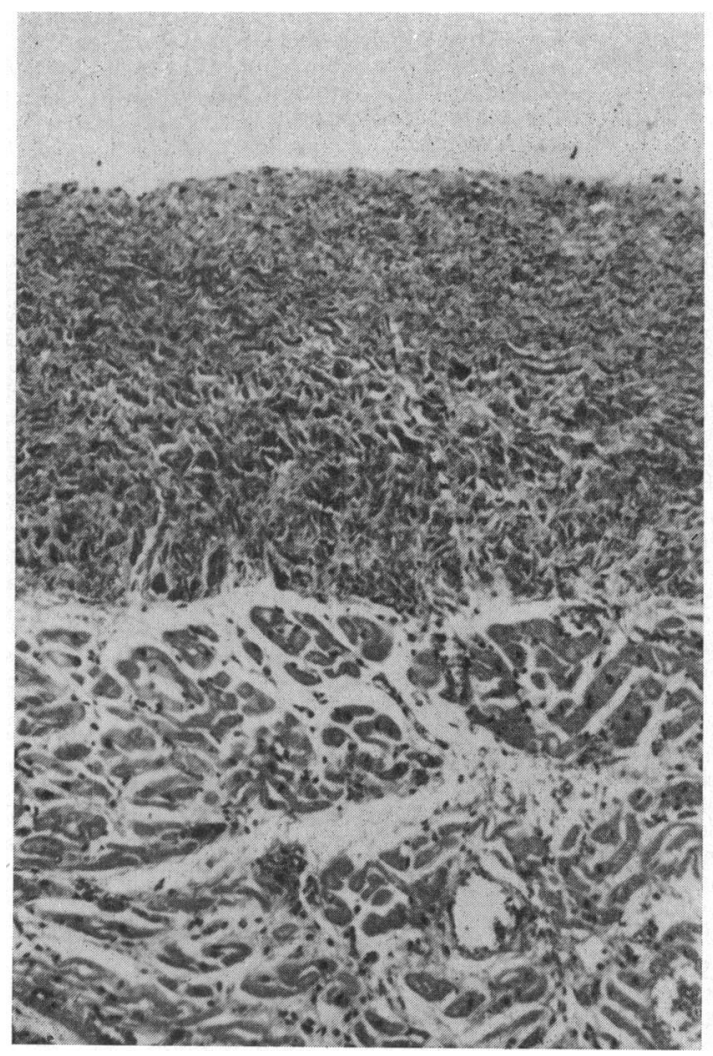

FIG. 2.-Left ventricular wall showing subendocardial fibrosis. The right half is thickened fibrotic endocardium. (van Gieson. $\times 27$.) 


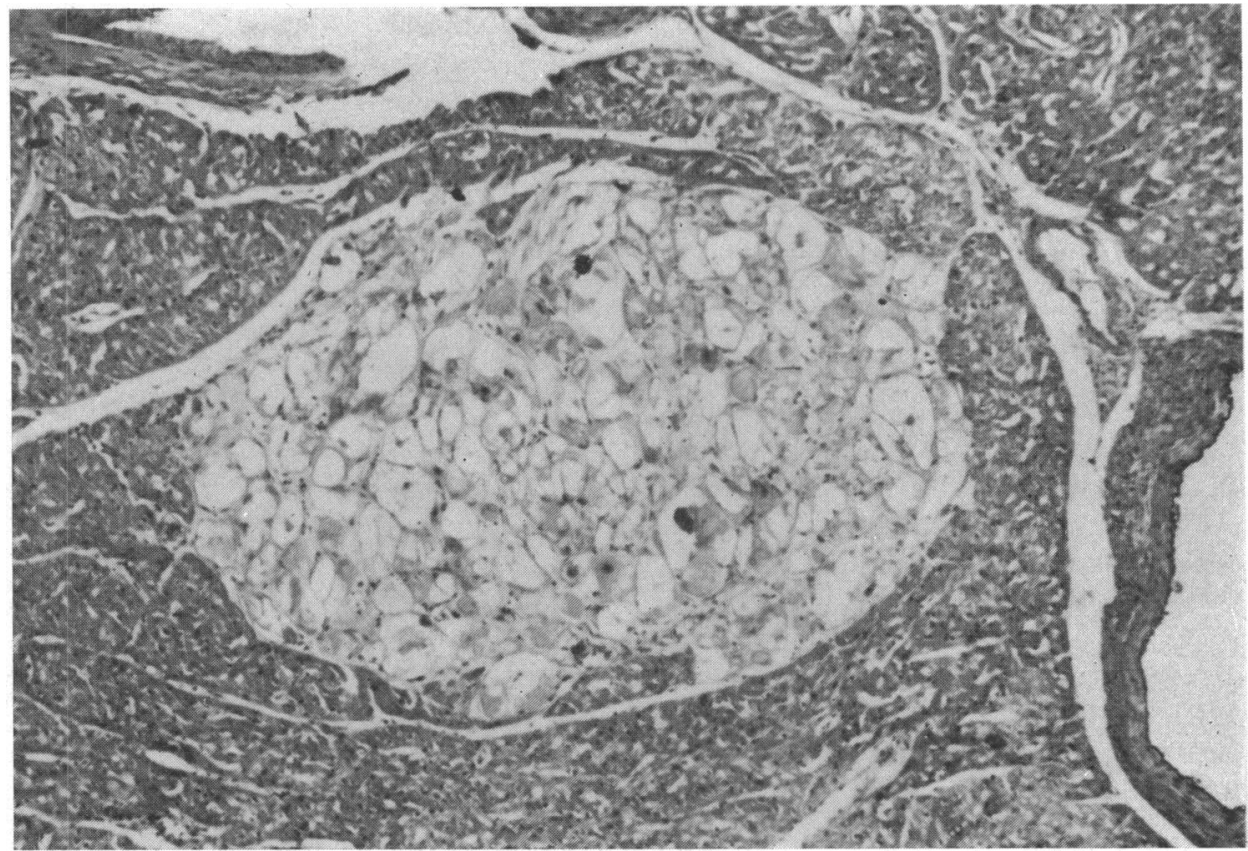

FIG. 3.-Case 1. Left ventricular wall showing a "rhabdomyoma". (Hæmatoxylin and eosin. $\times 40$.)

TABLE

Data on 62 infants with Congenital heart Disease. Ages at Death Ranging from 2 Days to 27 Months

\begin{tabular}{|c|c|c|c|c|}
\hline \multirow{2}{*}{ Type of basic malformation } & \multirow{2}{*}{ No. } & \multicolumn{3}{|c|}{ Condition of myocardium } \\
\hline & & $\begin{array}{c}\text { Normal apart } \\
\text { from hypertrophy }\end{array}$ & $\mid \begin{array}{c}\text { Minor degenerative } \\
\text { changes* }\end{array}$ & $\begin{array}{c}\text { Severe degenerative } \\
\text { changes } \dagger\end{array}$ \\
\hline 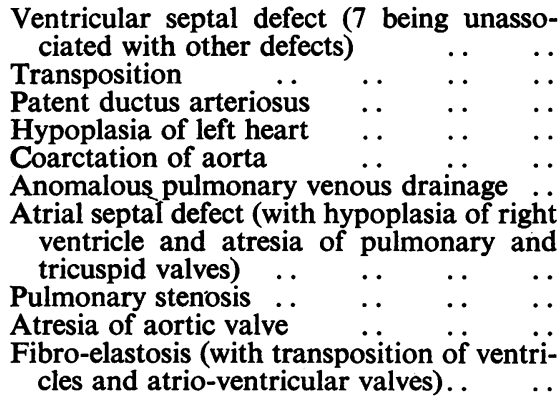 & $\begin{array}{r}38 \\
9 \\
2 \\
5 \\
2 \\
2\end{array}$ & $\begin{array}{l}13 \\
\frac{6}{1} \\
= \\
= \\
= \\
-\end{array}$ & $\begin{array}{r}22 \\
3 \\
1 \\
4 \\
2 \\
2 \\
\\
-1 \\
1 \\
1\end{array}$ & $\begin{array}{l}\frac{3}{1} \\
=\end{array}$ \\
\hline $\begin{array}{llll}\text { Total } & . . & . . & \end{array}$ & 62 & $\begin{array}{l}20 \\
(32 \%)\end{array}$ & $\begin{array}{c}37 \\
(60 \%)\end{array}$ & $\begin{array}{c}5 \\
(8 \%)\end{array}$ \\
\hline
\end{tabular}

* Ranging from subendocardial fibrosis without myocardial degeneration to mild patchy acute necrosis and/or fibrosis.

$\dagger$ Including more extensive necrosis and fibrosis, calcification, infarcted muscle, and "rhabdomyomata".

† This heart had been surgically explored. 
showed fibre hypertrophy in 40 cases, no hypertrophy in 20 cases; 2 were not examined. The right ventricle showed fibre hypertrophy in 54 cases, no hypertrophy in 8 .

Fatty Change. The left ventricle showed evidence of fatty change in 19 cases, no change in 40 cases; 3 were not examined. Of the 19 showing lipoid accumulation, 16 were associated with cyanosis; the other 3 were not cyanosed. Twenty-five cyanosed cases were unassociated with fatty change in the left ventricular myocardium, the remaining 3 cyanosed cases not being examined.

The right ventricle showed fatty change in 9 cases, no change in $22 ; 31$ were not examined. Of the 9 showing lipoid accumulation, 8 were associated with cyanosis.

Necrosis and Fibrosis. As can be seen, only 5 cases showed gross degenerative changes. Of these, one had been surgically explored and so was excluded from consideration; of the remaining 4, 3 had ventricular septal defects as well as other abnormalities, and 1 had a patent ductus arteriosus.

The four cases are summarized below.

Case 1. The child lived for 12 weeks and had been cyanosed from 7 weeks. The heart was enlarged with hypertrophied ventricles, a ventricular septal defect, and a truncus. Microscopical examination showed five small "rhabdomyomata" scattered in the ventricular myocardium, three in the right and two in the left (Fig. 3). There was much streaky myocardial fibrosis in the septum mostly involving the left ventricular side, and a little fibrosis elsewhere in the left ventricle, as well as a few small patches of acute necrosis.

Case 2. The child lived for five months and was acyanotic. The heart was enlarged with hypertrophied ventricles, two ventricular septal defects, overriding aorta, patent ductus, and a bicuspid pulmonary valve. Microscopical examination revealed moderate myocardial fibrosis throughout the septum (Fig. 4) with a few areas of calcification (Fig. 5) and scattered fibre necrosis. Elsewhere in the left ventricle there were a few areas of acute necrosis and some fibrosis. The right ventricle showed mild patchy myocardial fibrosis.

Case 3. The child lived for 13 months and was cyanosed. The heart was enlarged with hypertrophied ventricles, the cavity of the left ventricle being slit-like. A ventricular septal defect was present with a common atrio-ventricular canal,

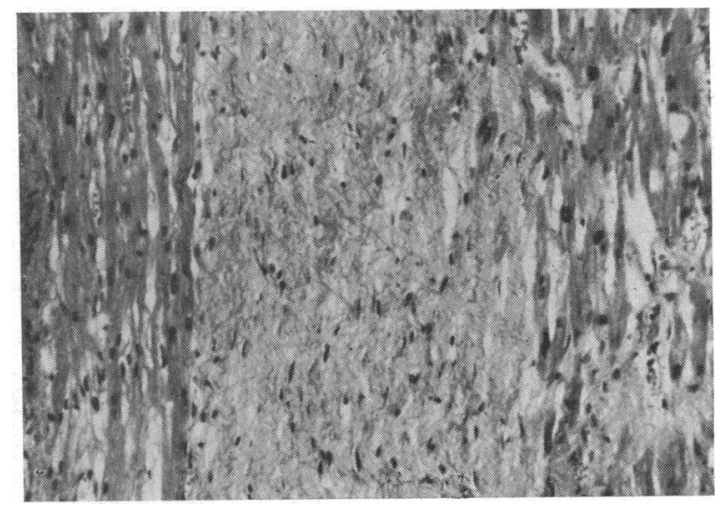

Fig. 4.-Case 2. Interventricular septum. The central part shows paler staining fibrous tissue. The myocardial fibres on each side are hypertrophic and have large, hyperchromatic nuclei. (Hæmatoxylin and eosin. $\times 46$.)

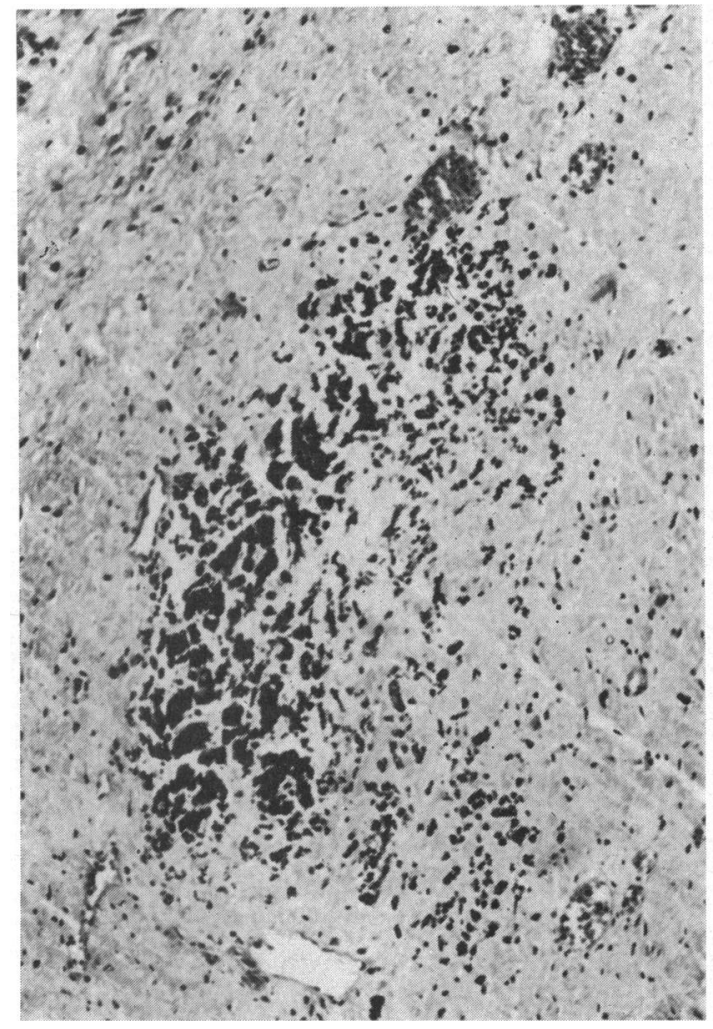

Fig. 5.-Case 2. Interventricular septum showing calcification in fibrous tissue. (Hæmatoxylin and eosin. $\times 63$.) 


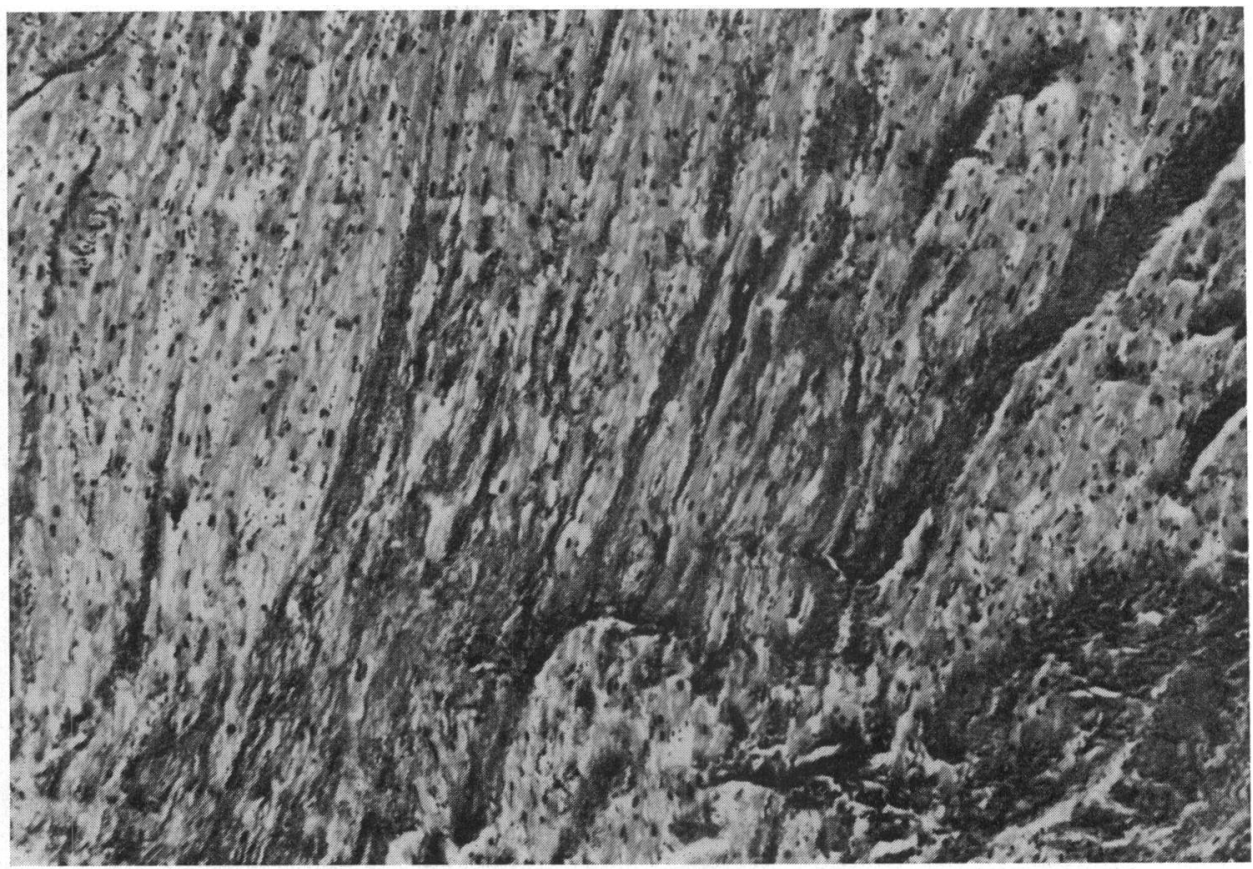

Fig. 6.-Case 4. Left ventricular wall showing infarction. The darker areas represent fibrous tissue. (van Gieson. $\times 35$.)

the major vessels were transposed, the left atrium was small, and the septum primum showed a few small perforations. Microscopically, the right ventricular half of the septum showed areas of acute necrosis, fibrosis, and one small area of calcification. Elsewhere in the right ventricle there were scattered areas of necrosis and some fibrosis.

Case 4. This child lived for 15 months and was slightly cyanosed. The heart showed dilated and hypertrophied ventricles in association with a patent ductus arteriosus. Microscopic examination showed patchy myocardial fibrosis in the left ventricular wall, a few areas of infarcted muscle (Fig. 6), one small area of calcification, and some endocardial fibrosis. One small area of infarction and early fibrosis was present in the right ventricle.

\section{Discussion}

Taussig (1960) describes the association of aberrant origin of the left coronary artery from the pulmonary artery with infarction and scarring of the left ventricular myocardium. Other than this, she makes no mention of myocardial degeneration and fibrosis in congenital cardiac malformations. Coleman and MacDonald (1962) describe a case of intrauterine myocardial infarction in which no right ventricular muscle was present, its place being taken by granulation tissue. No other anomalies were associated, and though the coronary vessels were normal, the authors were of the opinion that the infarction resulted from occlusion of the right coronary artery during gestation. The case then is perhaps of doubtful relevancy to the present context.

In this investigation interest was centred on the possibility of lesser degrees of, and perhaps progressive, irreversible myocardial degeneration. Particular search was therefore made for the presence of fibrosis and necrosis in the ventricular myocardium. Fibrosis could be considered, first, as evidence of aberrant differentiation of cardiac blastema during the course of fœtal development, a change concurrent with the main structural malformation. Secondly, fibrosis may be considered as evidence of repair in previously healthy myocardium, a change probably caused by cir- 
culatory defects within the heart induced as a later secondary effect of the main malformation. The presence of necrosis of muscular tissue can be interpreted more reliably as evidence of the same circulatory deficiency.

The results (see Table) were somewhat surprising because contrary to our expectation very little in the way of significant myocardial degeneration has been found. Twenty cases $(32 \%)$ showed a normal myocardium (i.e. apart from hypertrophy); $37(60 \%)$ showed minor changes ranging from slight endocardial fibrosis to mild patchy acute muscle necrosis alone or associated with fibrosis. Five hearts only $(8 \%)$ showed gross changes, and one of these was excluded from further consideration as it had been surgically explored.

Apart from hypertrophy, which is present to varying degrees in almost every case, there is no evidence to suggest that congenital cardiac malformations are associated with progressive irreversible degenerative changes in the myocardium in any significant number of cases. Thus from the point of view of surgical correction, the heart muscle appears to be in a fit state in the majority of cases, at least as far as can be gauged from histological assessment. Attempts at correlation between the type of congenital lesion and the severe degenerative changes were not possible in view of the small number of cases which showed marked pathological change. Since in a small number of cases marked myocardial degeneration does occur, and since from this study the type of structural lesion likely to be associated is unpredictable, it would be best to attempt surgical correction as soon as possible, quite apart from other considerations and indications.

Provided the structural or mechanical defects are compatible with life, survival then may become dependent to some extent on the nutrition of the myocardium, and when its function is impaired it may then fail and cause death before much necrosis or actual infarction can occur, the time between the final decompensation and death being too short for morbid anatomical changes to develop.

\section{SUMMARY}

Histological examinations were carried out on 62 structurally malformed hearts for evidence of irreversible degenerative changes. Of these, 37 showed minor pathological appearances not considered to be of any significance. Only 5 of the hearts showed gross changes, and one of these had been explored surgically. The other 4 showed necrosis and fibrosis in the interventricular septum as well as elsewhere in the ventricular walls. Because of the smallness of the series it was concluded that it was not possible to predict the type of abnormality most likely to be associated with gross degenerative changes in the myocardium and that in any case the percentage affected was so small as not to influence the time of surgical correction.

I wish to thank Dr. Malcolm Fowler for his encouragement and criticism; Mrs. Helga Thiede for the histological preparations; and Mr. Ray Boyd for the photographs.

\section{REFERENCES}

Coleman, E. N., and MacDonald, A. M. (1962). Intrauterine myocardial infarction. Arch. Dis. Childh., $37,444$. Coppoletta, J. M., and Wolbach, S. B. (1933). Body length and organ weights of infants and children. Amer. J. Path., 9, 55.

Gould, S. E. (1960). Pathology of the Heart, 2nd ed. Thomas, Springfield, Illinois.

Taussig, H. B. (1960). Congenital Malformations of the Heart, 2nd ed. Harvard University Press, Cambridge.

\section{ADDENDUM}

A further 43 congenitally malformed hearts not included in the above series had been examined histologically in the course of routine necropsies. However, only small sections of myocardium, prepared from blocks of more conventional size, were available in these cases. Of these, 40 were described as being normal or as showing fibre hypertrophy and/or fatty change. Only 3 showed irreversible degenerative changes, and one of these had been operated upon. Of the remaining 2 , the left ventricle of one showed areas of recent acute myocardial necrosis near the endocardium, fibre hypertrophy, and a little fatty change: this heart had a bicuspid, moderately stenosed aortic valve in 
association with mild preductal coarctation of the aorta and dilated hypertrophied ventricles. The child had lived for 23 days and was mildly cyanosed over the last three. The other case was one of complete anomalous pulmonary venous drainage into the coronary sinus in association with a dilated hypertrophied right heart and a small left heart. This child lived for five weeks and was cyanosed over the last three days. The myocardium showed, histologically, numerous small areas of muscle necrosis infiltrated by neutrophils. 